\title{
Solid State Interfaces: Toward an Atomistic-scale Understanding of Structure, Properties, and Behavior
}

\author{
D.L. Medlin, M.J. Demkowicz, and E.A. Marquis
}

We often imagine solid-state interfaces schematically as rather simple, two-dimensional, and compositionally abrupt entities. In any materials journal one will find numerous diagrams depicting boundaries between dissimilar materials or different grains as straight, flat lines. While such schematics are often a convenient and necessary simplification, it is important to remind oneself that reality is far from this ideal. Indeed, the properties and behavior of interfaces are fundamentally controlled by structures and compositional distributions that may have great complexity in three-dimensions at the atomic- and meso-scale.

While the importance of the atomicscale structure and composition of interfaces has been appreciated for decades, our ability to explore interfaces in their full, rich complexity has been limited. This situation has begun to change in recent years, with tremendous advances in theoretical and experimental ability to investigate interfaces. Highend computational capabilities are now enabling detailed atomistic simulations of interfaces and their dynamic behavior. In parallel, advances in microscopy, including atom probe tomography and aberration corrected transmission electron microscopy, are enabling interfacial analyses at unprecedented levels of spatial resolution and compositional sensitivity, increasingly in three dimensions.

These advances are providing new opportunities and needs for close interactions between the materials computation and microscopy communities. To this end, the TMS 2010 Annual Meeting featured a special symposium titled Solid State Interfaces: Toward an Atomistic-scale Understanding of Structure, Properties, and Behavior through Theory and Experiment. This symposium, which was jointly sponsored by the TMS Electronic, Magnetic, \& Photonic Materials Division, TMS Structural Materials Division, and TMS Chemistry and Physics of Materials Committee, featured over 50 talks and a joint session with the Vasek Vitek honorary symposium on Crystal Defects, Computational Materials Science and Applications.

This $J O M$ topic features papers by several of the participants at this symposium. C. Colliex et al. introduce the current state-of-the-art in high resolution scanning transmission electron microscopy (STEM), focusing on atomically resolved electron energy loss spectroscopy measurements at interfaces and applications of such analyses to epitaxial, oxide heterostructures.

G. Schmitz et al. explore the opportunities for analyses of interfacial chemistry being provided now by atom probe tomography. Examples in their overview include the analysis of interfacial compositional width in metallic multilayers, segregation to grain boundaries and triple junctions, and how such segregants can control the stability of nanocrystalline materials.

R. Srinivasan et al. emphasize the value of combining complementary information from techniques such as high resolution STEM and atom probe tomography. They present examples from analyses of $\gamma / \gamma^{\prime}$ interfaces in nickel-base superalloys and the $\alpha / \beta$ interface in $\beta$-titanium alloys, and discuss the connection of these results to mechanisms controlling microstructure evolution and stability.

Rahul K. Rajgarhia and coauthors continue this theme of microstructural stability. They review the mechanisms by which dopant segregation can stabilize nanostructures, presenting molecular dynamics simulations of three dimensional polycrystalline nanostructures aimed at explaining how antimony doping stabilizes nanocrystalline copper.

Finally, X. Zhang et al. present an overview of recent experimental and theoretical work investigating the enhanced radiation tolerance of metallic multilayer structures under helium irradiation conditions. They focus in particular on immiscible face-centered cubic/body-centered cubic interfaces, especially $\mathrm{Cu} / \mathrm{V}$, presenting both TEM analyses and atomistic simulations.

These papers illustrates the importance of atomic-scale modeling and experimental characterization to understanding solid-state interfaces in all their rich, 3-D complexity. The emerging capabilities in this area are presenting new scientific challenges and technological opportunities.

Douglas Medlin is principal member of the technical staff at Sandia National Laboratories, Michael Demkowicz is John C. Chipman Assistant Professor at the Massachusetts Institute of Technology, and Emmanuelle Marquis is an assistant professor at the University of Michigan, Ann Arbor. All are guest editors of the Chemistry and Physics of Materials Committee of the Electronic, Magnetic \& Photonic Materials Division of TMS.

\section{D.L. Medlin, M.J. Demkowicz, and E.A. Marquis are TMS Members!}

To read more about them, turn to page 9. To join TMS, visit www.tms.org/Society/Membership.aspx. 